\title{
A Bayesian Network Framework for Operations of Circuit Breakers
}

\author{
Alberto Carboni, Francesco Amigoni \\ $D E I B$ \\ Politecnico di Milano \\ Milan, Italy \\ alberto.carboni@outlook.com \\ francesco.amigoni@polimi.it
}

\author{
Khaled El Shawarby \\ DENG \\ Politecnico di Milano \\ Milan, Italy \\ khaled.elshawarby@polimi.it
}

\author{
Enrico Ragaini, Gabriele Perrone \\ Electrification Products \\ ABB S.p.A. \\ Bergamo, Italy \\ $\{$ enrico.ragaini, \\ gabriele.perrone\}@it.abb.com
}

\begin{abstract}
The digitalization of the electric grid is a major trend in today's energy industry and, especially with smart grid implementations, it is driving the innovation process in the whole sector. In particular, advanced analytics applications, e.g., prognostics, decision systems, and energy efficiency management, are gaining more and more importance for the involved players.

This paper proposes a framework for the operation and support of circuit breakers during their entire operational life, i.e., components replacement and maintenance strategies. This is done by means of tools from the field of artificial intelligence, i.e., Probabilistic Graphical Models (PGMs). PGMs provide an easy and intuitive way to model dependencies and causalities, thus, an actionable solution for companies operating on the field.

Index Terms-Probabilistic Graphical Model (PGM), Bayesian Network (BN), Decision Network (DN), Circuit Breaker (CB)
\end{abstract}

\section{INTRODUCTION}

One of the strongest trends in today's competitive landscape is digitalization, and industrial companies that rely on assets are facing new challenges in finding new algorithms and models to extract useful insights from the big amount of data collected on the field [1]. In this respect, the energy sector makes no exception and this transformation finds one of its best definitions in the smart grid concepts [2]. The digitalization offers new opportunities to the involved players, opportunities that are likely to become a need in the years to come. In particular, advanced analytics applications for enhancing reliability, productivity and planning are extremely hot topics [3]-[5].

When dealing with insights and analytics applications it is fundamental to ensure their sustainability at company level [6]. The applications, in fact, have to be, first, easily integrated in existing processes and products, and, second, easy to use. Furthermore, given the nature of the applications, the framework on which they are built should be able to integrate additional company-specific insights and expertise. For all these reasons, a particular field of Artificial Intelligence (AI), i.e., Probabilistic Graphical Models (PGMs), seems particularly promising for employment in these scenarios [7], [8].

The results reported in this paper are part of the activities of the ABB \& Politecnico di Milano Joint Research Centre.

Alberto Carboni is now with the MBDA Systems and Safety Design Engineering Department.
A PGM is a declarative representation of a real world model. PGMs may refer to either Bayesian Networks (BNs) or Markov Networks (MNs). The use of BNs in modelling and analysis of complex systems has gained in the last few years more and more interest [8]. This is due to the great advantage of PGMs over other AI frameworks, i.e., the human readability of the resulting models and the efficient inference processes.

This work proposes a BN-based framework for the optimal operation and support of Circuit Breakers (CBs) during their operational life.

CBs are an extremely critical elements in an electric system and a lot of research has been done to better understand the ageing of $\mathrm{CBs}$ for maintenance optimization and reliability assessment [9]-[11].

In general, because of the difficulties to create theoretical models describing the failure modes in $\mathrm{CBs}$, data-driven approaches have always been of great interest, in particular in their probabilistic versions [12], [13].

The framework proposed in this paper is composed of:

- A BN to be used during the inspection of CBs, i.e., a support system to decide which elements to prioritize first, starting from the elements with higher fault probability rather than from the less critical ones.

- A Decision Network (DN), i.e., a BN with random variables as well as decision nodes, that exploits probabilistic information in a decision making process by following the Maximum Expected Utility (MEU) principle [14], [15]. The aim is to support the operator in deciding whether or not to replace a $\mathrm{CB}$ or update only few components. This, because of the serious implications from both a technical and economic points of view, implies the possibility to plan potentially years ahead the complete or partial replacement of $\mathrm{CBs,} \mathrm{with} \mathrm{clear}$ advantages.

The potential benefit of the framework proposed in this paper can be clearly understood if it is considered the definition of Reliability Centered Maintenance (RCM) according to [16]:

$\mathrm{RCM}$ is used to determine what failure management strategies should be applied to ensure a system achieves the desired levels of safety, reliability, en- 
vironmental soundness, and operational readiness in the most cost-effective manner.

RCM aims at enhancing system dependability, i.e., the behavior of a system, in terms of reliability, availability, maintainability, safety and security.

The proposed method has the objective of being a support to management strategies definition and, in doing that, overcoming the main limitations of classical approaches to dependability modeling and analysis [17]-[19].

The rest of this paper is organized as follows: Section II deals with the definition of the BN describing a CB. Section III is a preliminary proof of concept of the proposed method starting from data publicly available; for the inference and modelling part an open-source Python library has been used [20]. Finally, Section IV concludes the paper.

The choice of not disclosing any company-specific data is done because of the confidential and strategic nature of the data involved.

\section{A Naive Bayesian Model for Circuit Breakers}

\section{A. Failures in Circuit Breakers}

The choice of not disclosing company-specific data because of their confidential and strategic nature means that, in this work, publicly-available data have been used. The considered dataset is the result of a data collection campaign done by the European Distribution System Operators (DSOs) [21].

In particular, the circuit breakers of interest are all the single-pressure $S F_{6}$ CBs placed in service during the period 01/01/1983 - 01/01/1992, installed outdoor in non-metal enclose, spring operated, with a rated voltage $V_{n}=63-100$ $\mathrm{kV}$.

In [21], two different types of failures are defined, i.e., major and minor failures. A major failure is a complete failure of the $\mathrm{CB}$ with the loss of one or more of its fundamental functions. On the contrary, a minor failure is a failure that does not imply the loss of a fundamental function.

For both the minor and major failures the relevant failure rates have been calculated. The failure rate for a specific component $x_{i}\left(F R_{x_{i}}\right)$ is defined as the ratio between the number of failures and the considered time interval (failures/time) weighted by the percentage of faults due to $x_{i}$ over the entire population of the report $\left(p_{x_{i}}\right)$. It is important to notice that the entire population of the report, i.e., all the Medium Voltage (MV) $\mathrm{CBs}$, is larger than the considered subset of $\mathrm{CBs}$, i.e., the single-pressure $S F_{6} \mathrm{CBs}$ placed in service during the period 01/01/1983 - 01/01/1992, installed outdoor in non-metal enclose, spring operated, with a rated voltage $V_{n}=63-100$ $\mathrm{kV}$. The component-specific failure rate is, thus, calculated as:

$$
F R_{x_{i}}=p_{x_{i}} \cdot \frac{\text { failures }}{\text { time }}
$$

The considered components or subsystems are the following: making and breaking units (MBU), auxiliary interrupters and resistors (AIR), main insulation to earth (MIE), tripping and closing circuits (TCC), auxiliary switches and drives (ASD), contactors, relays, heaters, fuses, thermostats (CRH),
TABLE I

LIST OF CIRCUIT BREAKER COMPONENTS AND THEIR MAJOR AND MINOR FAILURE RATES

\begin{tabular}{|c|c|c|}
\hline \multicolumn{3}{|c|}{ Failure rates [failure/circuit-breaker-year] } \\
\hline Component & Major Failure & Minor Failure \\
\hline MBU & 0,0015 & 0,0021 \\
\hline AIR & 0,0001 & 0,0001 \\
\hline MIE & 0,0006 & 0,0046 \\
\hline TCC & 0,0011 & 0,0003 \\
\hline ASD & 0,0008 & 0,0005 \\
\hline CRH & 0,0008 & 0,0012 \\
\hline GDS & 0,0004 & 0,0024 \\
\hline CMP & 0,0015 & 0,0041 \\
\hline ESt & 0,0008 & 0,0016 \\
\hline CEl & 0,0010 & 0,0025 \\
\hline ADD & 0,0010 & 0,0011 \\
\hline MTr & 0,0004 & 0,0003 \\
\hline Other & 0,0007 & 0,0012 \\
\hline
\end{tabular}

gas density supervision (GDS), compressor, motors, and pump (CMP), energy storage (ESt), control elements (CEl), actuator and damping device (ADD), and mechanical transmission (MTr). All the other elements and subsystems have been aggregated together because either their individual failure rates are too small alone or there are not enough data to characterize them.

Table I shows the failure rates for each component divided into major and minor failures.

\section{B. Naive Bayesian Model}

The definition of a descriptor for a real-world CB is of paramount importance for our application, and the model we chose is a $\mathrm{BN}$ in its naive implementation. A naive Bayesian model assumes that all the basic events in the model are independent.

Despite the strong independence assumption, the naive Bayesian models are largely used in applications where models are learned from data with a large number of features and a small number of instances [7]. A serious implication of the independence assumption that must be taken into account in the definition of the problem is the degradation in the diagnostic performance when the number of features increases. This is mainly due to violations of the conditional independence assumption [22].

Figure 1 shows an example of a naive $\mathrm{BN}$ : the nodes (ovals) are the model variables, while the edges correspond to direct probabilistic interactions. In the proposed application, the variables (events) are the components of the $\mathrm{CB}$, e.g., damping device, main insulation to earth, breaking unit. Each node represents a basic component, whose probability of being or not in a fault state are described by the well-known exponential distribution. Each variable is modelled as a Boolean one, i.e., the variable is either in an operational state (TRUE state) or in a failure state (FALSE state).

Table II shows the probability associated to a generic variable $x_{i}$ in the system of interest. The probability that the element $x_{i}$ is operational is described by an exponential distribution with parameter $\lambda$, the failure rate. The choice of 


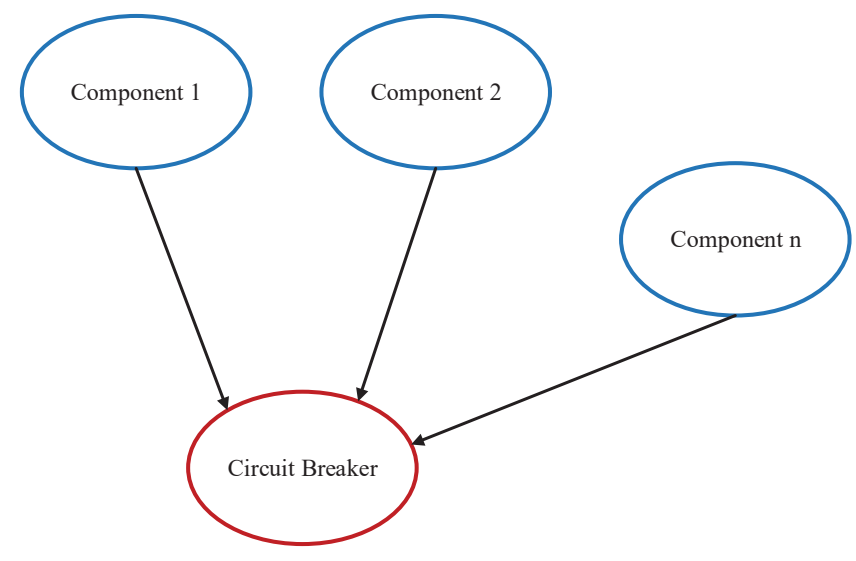

Fig. 1. Example of a naive Bayesian network

TABLE II

ELEMENT PROBABILITY DEFINITION

\begin{tabular}{|l|l|}
\hline $\mathbf{P}\left(\mathbf{x}_{\mathbf{i}}=\right.$ Working $)$ & $\mathbf{P}\left(\mathbf{x}_{\mathbf{i}}=\right.$ Failed $)$ \\
\hline$e^{-\lambda t}$ & $1-e^{-\lambda t}$ \\
\hline
\end{tabular}

the exponential distribution over the Weibull one is mainly driven by the data currently available to the authors. As new data are collected a Weibull-based approach will be used, as it proves to be more accurate [23].

Once that the failure probabilities for each element have been determined is possible to define the tabular Conditional Probability Distribution (CPD) for the entire system, in this case the CB.

Table III shows the resulting CPD. It is obtained as the truth table for an OR-gate with Common Cause Failure (CCF) [8], [24]. This means that if at least one component fails, then, the entire system fails. This is done for safety purposes since a failure in any part of a $\mathrm{CB}$ poses a serious threat to operators as well as electric equipments.

Furthermore, if all the components are working correctly the probability of a $\mathrm{CB}$ failure is not zero but $0.5 \%$. This is done because the complexity of the considered system is high and there is not enough information to describe all the possible dependencies and factors affecting the system, thus, in order to avoid the construction of a more complex and detailed model, a CCF is introduced.

Finally, another advantage of this type of modelling is in the limited size and complexity of the resulting BN. This means that exact inference algorithms can be employed. Exact inference algorithms, in fact, feature an exponential complexity in the size of the graph. In this work the Variable Elimination

TABLE III

CIRCUIT BREAKER NOISY-OR TABULAR CPD DEFINITION

\begin{tabular}{|c|c|c|}
\cline { 2 - 3 } \multicolumn{1}{c|}{} & All components $\mathbf{x}_{\mathbf{i}}$ are Working & At least one component $\mathbf{x}_{\mathbf{i}}$ is Failed \\
\hline $\mathbf{P}(\mathbf{C B}=$ Working $)$ & 0.995 & 0 \\
\hline $\mathbf{P}(\mathbf{C B}=$ Failed $)$ & 0.005 & 1 \\
\hline
\end{tabular}

(VE) is employed [7].

\section{Decision Network}

The decision process is implemented by integrating in the naive Bayesian model some decision elements. From a MEU point of view, this can be described by the following equations:

$$
\begin{aligned}
& U_{1}=P(C B=F) \cdot U(C B) \\
& U_{2}=P\left(C B=F \mid x_{i}=T\right) \cdot U(C B)+U\left(x_{i}\right)
\end{aligned}
$$

where $P(C B=F)$ is the probability that the $\mathrm{CB}$ is in failed condition, $U(C B)$ is the utility associated with the failure of the $\mathrm{CB}$, i.e., the economic value of the fault. $P\left(C B=F \mid x_{i}=T\right)$ is the conditional probability that, given that the element $x_{i}$ is working correctly, the $\mathrm{CB}$ is in a failed condition. Finally, $U\left(x_{i}\right)$ is the utility associated with element $x_{i}$, i.e., the economic value associated with the replacement of the component $x_{i}$.

Therefore, Eq. (2) represents the expected utility associated with the fault of the CB. While Eq. (3) is the expected utility associated with the fault of the $\mathrm{CB}$, if component $x_{i}$ is replaced. It is important to notice that this could also mean (i) the replacement of the whole $\mathrm{CB}$ (ii) the replacement of more than one component.

Finally, the output of the decision process is calculated as:

$$
M E U=U_{1}-U_{2}
$$

A $M E U>0$ means that the replacement of the component is profitable while a $M E U<0$ means that, given the uncertainties involved, there is no reason to replace the considered element. Finally, a $M E U=0$ means that the impact of doing or not doing the replacement is the same.

\section{CASE Study}

Starting from the failure rates calculated in Section II-A and the model described in Section II-B, it is possible to calculate the major and minor failure probabilities. Fig. 2 shows the failure probabilities for a $\mathrm{CB}$ over a time interval equal to 50 circuit-breaker years, where $t=1$ circuit-breaker year is the installation year while $t=50$ circuit-breaker year is the decommissioning year.

Let us consider a CB operating on the field, that has been operational for 20 years and now it is in a failure state. The proposed framework can be used to do inference on the CB in order to define the components' failure probabilities and, hence, it helps the operator in prioritizing the most critical components. The inference to be done amounts to calculate the following probabilities:

$$
P\left(x_{i}=F \mid C B=F\right) \quad \forall i
$$

Table IV shows the resulting major and minor components failure probabilities for a 20 -year-old circuit breaker. The inspection procedure is defined by sorting this probabilities in a descending order, in doing so, the inspection procedure is customized on the $\mathrm{CB}$ of interest. 


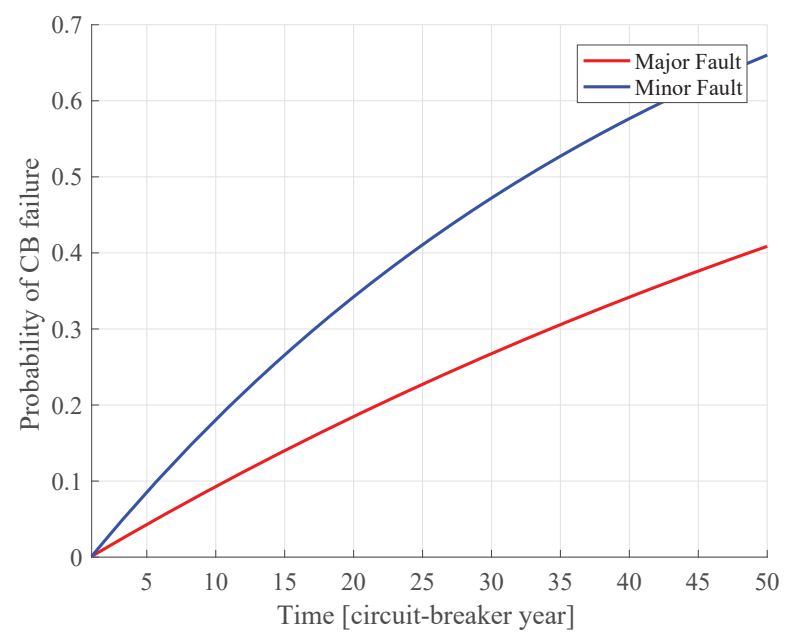

Fig. 2. Overall $\mathrm{CB}$ failure probabilities versus circuit-breaker years

TABLE IV

COMPONENTS FAILURE PROBABILITIES

\begin{tabular}{|c|c|c|}
\hline Component & Major Failure & Minor Failure \\
\hline MBU & 0.1528 & 0.1153 \\
\hline AIR & 0.0103 & 0.0056 \\
\hline MIE & 0.0617 & 0.2465 \\
\hline TCC & 0.1125 & 0.0168 \\
\hline ASD & 0.0820 & 0.0279 \\
\hline CRH & 0.0820 & 0.0665 \\
\hline GDS & 0.0412 & 0.1314 \\
\hline CMP & 0.1528 & 0.2208 \\
\hline ESt & 0.0820 & 0.0883 \\
\hline CEl & 0.1024 & 0.1368 \\
\hline ADD & 0.1024 & 0.0610 \\
\hline MTr & 0.0412 & 0.0168 \\
\hline Other & 0.0719 & 0.0665 \\
\hline
\end{tabular}

Fig. 3 shows the graphical representation of the sorted inspection procedure. The resulting inspection sequence, in the presence of an unknown failure, is obtained by summing up the probabilities for a major and minor failure and, then, sorting them in a descending order. In particular, we can isolate four main possible drivers in the $\mathrm{CB}$ failure, i.e., compressor, motors, and pump, main insulation to earth, making breaking units and control elements.

This kind of analysis, triggered by the failure of the $\mathrm{CB}$, is able to reduce downtime due to unexpected maintenance. In particular, it becomes useful for CBs whose elements have been replaced or repaired during their life, in this case, in fact, it is not so trivial to determine which element is more likely to be in a fault state.

Let us now consider that the aforementioned failed CB was installed in a production plant as the main $\mathrm{CB}$ for a production line. In such a plant, it is likely that similar CBs are installed as main protection for other production lines. Therefore, in the presence of a CB failure, there is interest in guaranteeing a safe operation for the other production lines and in understanding whether or not corrective actions should be taken to reduce the risk of other faults.
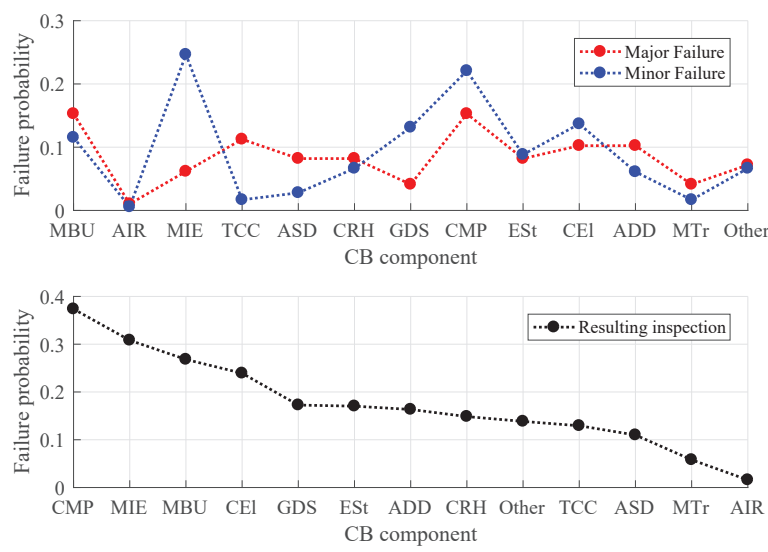

Fig. 3. Failure probabilities and resulting inspection list

TABLE V

CIRCUIT BREAKER COMPONENTS REPLACEMENT ASSOCIATED UTILITIES

\begin{tabular}{|c|c|}
\hline Component & Utility \\
\hline MBU & 5000 \\
\hline AIR & 4000 \\
\hline MIE & 4000 \\
\hline TCC & 3000 \\
\hline ASD & 3000 \\
\hline CRH & 2000 \\
\hline GDS & 2000 \\
\hline CMP & 2000 \\
\hline ESt & 2000 \\
\hline CEl & 1000 \\
\hline ADD & 1000 \\
\hline MTr & 1000 \\
\hline Other & 1500 \\
\hline
\end{tabular}

In order to do that, there is the need to calculate the utility associated with different replacement strategies, as in (2-3).

Table V defines a set of dummy utilities associated to the replacement of the $\mathrm{CB}$ components. The utility associated to a major failure are assumed to be equal to $100 k$-unit and $50 k$ unit for a minor failure.

Now, it is possible to calculate the utility for different replacement strategies. For the sake of clarity, in this case study, we have considered the replacement of only one component at a time but, in principle, more than one component could be replaced. Table VI shows the resulting MEUs for different replacement strategies in the presence of major and minor failures.

Fig. 4 is a graphical representation of the resulting MEUs. From the figure, it emerges that the most promising strategy for the mitigation of minor failures is the replacement of the main insulation to earth. However, it turns out that doing maintenance on the control elements and/or compressor, motors, and pump is the best strategy for the mitigation of both major and minor failures.

\section{CONCLUSION}

This work proposes an advanced analytics framework to support smart operation of circuit breakers throughout their 
TABLE VI

$M E U$ COMPARISON FOR DIFFERENT REPLACEMENT STRATEGIES

\begin{tabular}{|c|c|c|}
\hline Replaced Component & MEU major $_{\text {mEU }}$ & ME $_{\text {mor }}$ \\
\hline MBU & -2540 & -3620 \\
\hline AIR & -3830 & -3935 \\
\hline MIE & -3020 & 1350 \\
\hline TCC & -1200 & -2805 \\
\hline ASD & -1700 & -2675 \\
\hline CRH & -700 & -1220 \\
\hline GDS & -1350 & -420 \\
\hline CMP & 460 & 750 \\
\hline ESt & -700 & -955 \\
\hline CEl & 630 & 650 \\
\hline ADD & 630 & -285 \\
\hline MTr & -350 & -805 \\
\hline Other & -360 & -720 \\
\hline
\end{tabular}

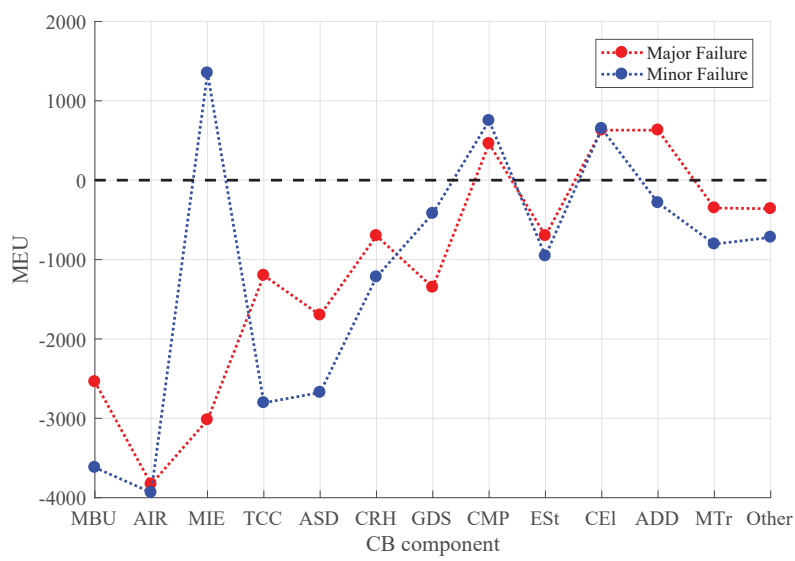

Fig. 4. Maximum Expected Utilities per type of failure per component

entire operational life.

The main advantage of the framework proposed is in its ease of reading and interpreting the knowledge it conveys; BNs, in fact, allows an intuitive definition of the structure of the probabilistic relationships underlying the model. Thus, the resulting output can be easily checked and understood by human operators.

Finally, it is important to notice that, even though this work focuses on $\mathrm{CBs}$, the proposed framework can be, in principle, scaled and reproduced for other systems of interest, e.g., power converters, data acquisition systems, PV plants, and many more.

\section{REFERENCES}

[1] B. Cheng, J. Zhang, G. P. Hancke, S. Karnouskos, and A. W. Colombo, "Industrial cyberphysical systems: Realizing cloud-based big data infrastructures," IEEE Industrial Electronics Magazine, vol. 12, no. 1, pp. 25-35, March 2018.

[2] H. Farhangi, "The path of the smart grid," IEEE Power and Energy Magazine, vol. 8, no. 1, pp. 18-28, January 2010.

[3] K. Wang, H. Li, Y. Feng, and G. Tian, "Big data analytics for system stability evaluation strategy in the energy internet," IEEE Transactions on Industrial Informatics, vol. 13, no. 4, pp. 1969-1978, Aug 2017.

[4] P. Lade, R. Ghosh, and S. Srinivasan, "Manufacturing analytics and industrial internet of things," IEEE Intelligent Systems, vol. 32, no. 3, pp. 74-79, May 2017.
[5] R. C. Basole, M. A. Bellamy, H. Park, and J. Putrevu, "Computational analysis and visualization of global supply network risks," IEEE Transactions on Industrial Informatics, vol. 12, no. 3, pp. 1206-1213, June 2016.

[6] D. Wang, "Building value in a world of technological change: Data analytics and industry 4.0," IEEE Engineering Management Review, vol. 46, no. 1, pp. 32-33, Firstquarter 2018.

[7] D. Koller and N. Friedman, Probabilistic graphical models: principles and techniques, 2009.

[8] P. Luigi and C. R. Daniele, Modeling and analysis of dependable systems: a probabilistic graphical model perspective. World Scientific, 2015.

[9] S. Turrin, B. Deck, M. Egman et al., "Medium voltage equipment monitoring and diagnostics: technological maturity makes concepts compatible with expectations," CIRED, June 2015.

[10] T. M. Lindquist, L. Bertling, and R. Eriksson, "Circuit breaker failure data and reliability modelling," IET Generation, Transmission Distribution, vol. 2, no. 6, pp. 813-820, November 2008.

[11] M. Scarpellini, K. Perdon, L. Cavalli, and M. Testa, "Innovative analytics to estimate the probability of failure and remaining useful life of medium-voltage breakers," CIRED - Open Access Proceedings Journal, vol. 2017, no. 1, pp. 2379-2382, 2017.

[12] T. Lindquist, L. Bertling, and R. Eriksson, "A feasibility study for probabilistic modeling of aging in circuit breakers for maintenance optimization," in 2004 International Conference on Probabilistic Methods Applied to Power Systems (PMAPS), Sept 2004, pp. 1020-1025.

[13] P. Westerlund, P. Hilber, T. Lindquist, and S. Kraftnät, "A review of methods for condition monitoring, surveys and statistical analyses of disconnectors and circuit breakers," in 2014 International Conference on Probabilistic Methods Applied to Power Systems (PMAPS), July 2014, pp. 1-6.

[14] J. Von Neumann and O. Morgenstern, Theory of Games and Economic Behavior. Princeton University Press, 1947.

[15] R. A. Howard and J. E. Matheson, "Influence diagrams," Decision Analysis, vol. 2, no. 3, pp. 127-143, 2005.

[16] "DOD 4151.22-M RELIABILITY CENTERED MAINTENANCE (RCM) ."

[17] W. G. Schneeweiss, "The fault tree method," 1999.

[18] S. Contini and A. Poucet, "Advances on fault tree and event tree techniques," in Systems Reliability Assessment. Springer, 1990, pp. $77-102$.

[19] R. A. Sahner, K. Trivedi, and A. Puliafito, Performance and reliability analysis of computer systems: an example-based approach using the SHARPE software package. Springer Science \& Business Media, 2012.

[20] A. Ankan and A. Panda, "pgmpy: Probabilistic graphical models using python," in Proceedings of the 14th Python in Science Conference (SCIPY 2015). Citeseer, 2015.

[21] I. C. on Large High Voltage Electric Systems. Study Committee 13 (Switching Equipment). Working Group 06 (Reliability of HV circuit breakers) and A. Janssen, Final report of the second international enquiry on high voltage circuit-breaker failures and defects in service. CIGRE, 1994

[22] A. Y. Ng and M. I. Jordan, "On discriminative vs. generative classifiers: A comparison of logistic regression and naive bayes," in Advances in neural information processing systems, 2002, pp. 841-848.

[23] W. R. Wessels, "Use of the weibull versus exponential to model part reliability," in 2007 Anпиal Reliability and Maintainability Symposium, Jan 2007, pp. 131-135.

[24] A. Mosleh, K. Fleming, G. Parry, H. Paula, D. Worledge, and D. M. Rasmuson, Procedures for treating common cause failures in safety and reliability studies: Procedural framework and examples. Newport Beach, CA (USA): Pickard, Lowe and Garrick, Inc., 1988. 